- - . - - مجلة علوم الرافني، المجلد 26، العدد 1، ص74 -85، 2017 - - - - -

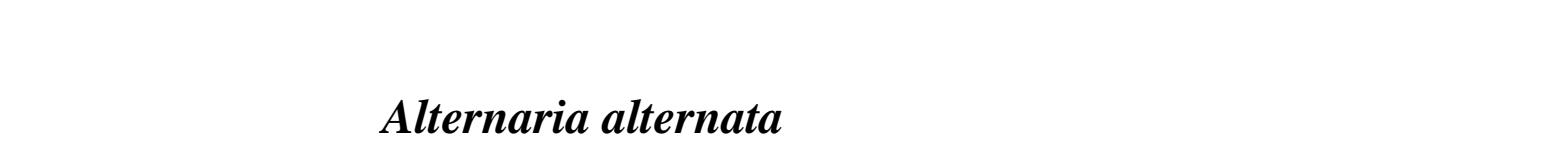

\author{
هان وليد هامي \\ قنم علوم حيلة/كلية العلوم/جلمعة الموصل

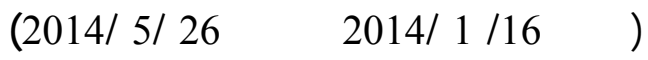

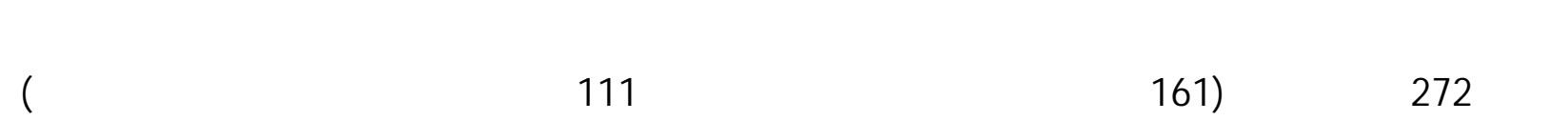
توزعت هذه العزلات بعضها كطافرات مقاومة للمواد الكيميائية الاتية التوبلس Topas والازلكوانين 8-Azaguanine والكلورل

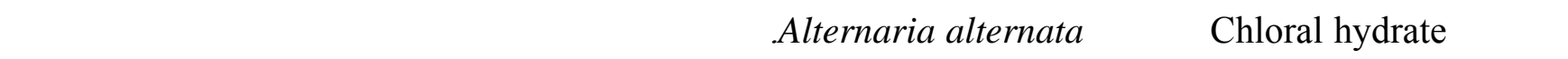
التركيز المثط الانف للمواد الكيميائية. وقد كلت فترات التعريض 20 و40 و60 و90 دقيقة والتركيز المثط الانف 150 ميكروغرل/dل للتوبلس و3.5 ميكروغراd /لمل للازلكوانين و 4.5 ملي مولر للكلورل هايدريت. وجرى هسلب تكرار الطافرات

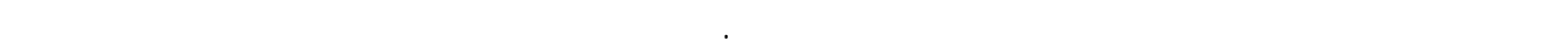

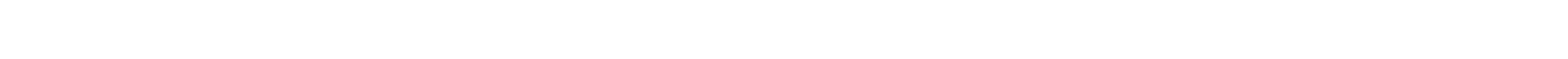

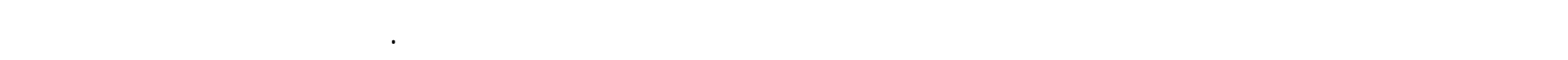

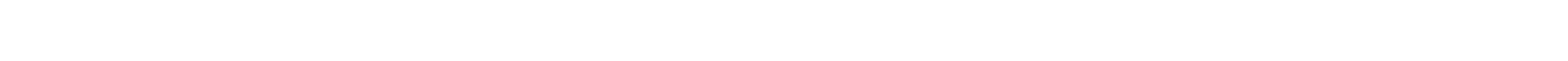
هايدريت وبنسب مئوية مرقفعة اذ بلغت 83 \% بين التوبلس والكلورل هايدريت و93.2 \% بين التوبلس والازلكولنين، في حين

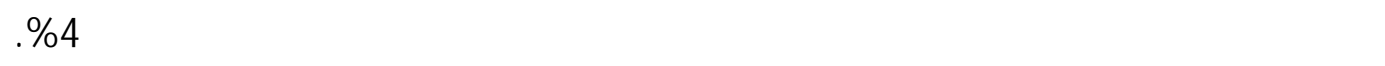

الكالت الدالة: Alternaria alternata ،طفورات مقاومة، لشعة اللثهس، مقاومة كرضية، مواد كيميائية.

\title{
Isolation of Mutants Resistant to some Chemicals from Sunlight-Exposed and Unexposed Samples of the Fungus Alternaria alternata
}

\author{
Huda W. Hadi \\ Department of Biology/ College of Science/ University of Mosul
}

\begin{abstract}
A total of 272 mutants (161 from sunlight exposed and 111 from unexposed conidia) resistant to the chemicals Topas, 8-azaguanine or Chloral hydrate were Isolated in the fungus Alternaria alternata. Initially, the exposure periods to sunlight and the minimal inhibitory concentrations for the chemicals were determined. The exposure periods were 20, 40,60 and 90 minutes and the minimal inhibitory concentrations were $150 \mu \mathrm{g} / \mathrm{ml}$ for Topas, $3.5 \mu \mathrm{g} / \mathrm{ml}$ for 8 -azaguanine and $4.5 \mathrm{mM}$ for Chloral hydrate. The frequency of mutants resistant to each chemical was calculated and although the frequencies from the exposed samples were greater than those from unexposed one, the differences, however, were
\end{abstract}




$$
\text { هلsى وليد هادي }
$$

statistically not significant pointing to the weakness of mutagenicity of the sunlight in the present experimental protocol. Cross resistance exhibited by any of the three classes of resistant mutants to other two chemicals was also tested. A fraction of $93.2 \%$ of the Topas resistant mutant cross resisted 8azaguanine as well, and $83 \%$ of them cross resisted Chloral hydrate. Azaguanine resistant mutants and Chloral hydrate resistant mutants, however, exhibited very low cross resistance to those two chemicals, being no more than $4 \%$ as its higher proportion.

Keywords: Alternaria alternata, resistant mutants, sunlight, cross resistance, chemicals.

\section{قملف}

يعد الفطر Alternaria alternata لحد لجنالس الفطريت الخطية ذات الابواغ المقنمة ولسعة الانتشار اذ يعد من لكبر المسببت المرضية للمحاصل الزراعية سواء في الهقل او اثناء القل او الخزن فهو يصيب عدداً كبيراً من النواع الفولكه

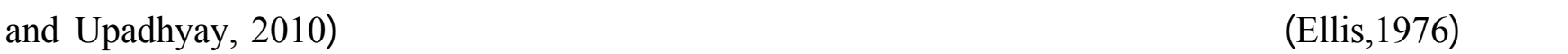
.(Prasad

لن لستعمل المبيدات الكيميائية في للسيطرة على هذا الممرض والفطريلت الممرضة النباتية الخرىىطريقةسريعة التأثير واقتصاية لكونها ت تؤدي دورَ كبيرَ في وقاية النباتلت او حماية المحاصل من الاصابة او الحد من النتار الهشرات النقلة للأمراض النباتية (العال، 2006) لكن في الوقت فغه فلن الاستعمل المتكرر لهذه الكيمياويت يمكن لن يحث التغيرت الطبية الطبيعية للجينا من خلال نشوء الطفرات المقاومة في تلك المجلمبع الفطرية وبالتالي سيصعب للسطرة على هذه الممرضلت مسنقبلا بلستعمل هذه المبيدك نفسها فتصح هذه الآفلت ذات ضراوة عالية (Moore and Frazer , 2002). الطفرة المقاومة هي طفرة لملمية Forward tionmuta كتسبها الكائن تحت طظروف ليست منلسبة للنهط البري (al.,1979

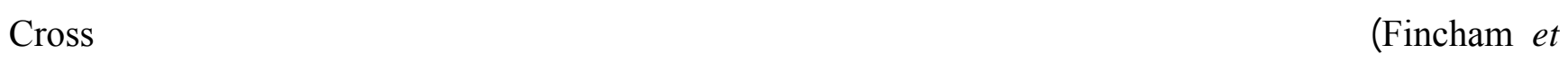
rusistance المختلفة كيميائيا (Leroux et al., 1999). لذا تجررى محاولات كثيرة لتصنيع مبيدات جديدة ضد هذا الفطر و الفطريك الممرضة النباتية الخرى مل Fusarium و Septoria و Helminthosporium ليس لها تأثيرك سلبية على المحاصيل الزراعية من

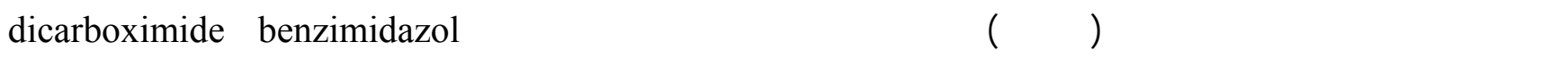

. (Gehmann et al.,1990) guanidine 2-(4-thiazolyl)-11-1-benzimidazoled المادة الفعرون 1991 المبيد توبلس Topas صيغتها العلمة (C10H7N3S) والإم العلمي له Thiabendazole وهو مبيد فطري جهازي يتدلخل في عملية تتمبع خيوط المغزل اثناء عملية الاقسلم الخلوي B tubulin assembly يم لمتعمل هذا المبيد ضد ممرضلت نباتية مختلفة مل النواع Fuspergillus والملثية(Harding, 1980) وبستعل لتزويد وقاية كلملة 100\% ضد مسببت البياض الدقيقي 2010) 1,1,2-2). (Reuveni et al., 1998; trichloroethylene وهو ومركب هيدروكربوني يستعل في الصناعلت الدوائية كمسكن كما يستعل كمادة فعالة يخل في تركيب العديد من المبيدات الهشرية والعشبية مل DDT, trichlorofon, dichlorovos, trichloroacetic الخ (IARC,1995). كما

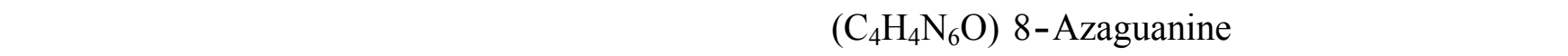


الحمض النووي مقوص الاوكسجن (DNA) قالب للانجشار في تركيبه ولحداث الخل فيه (Smith and Harrill,1968) اذ

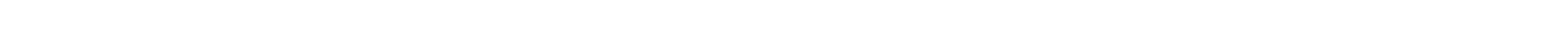
كافت بيورين او بيرمدين (Pantazopoulou and Diallinas, 2007)، يوضح اللثكل (1) الصبغ البنائية للمواد قيد الاختبار.

Azaguanine

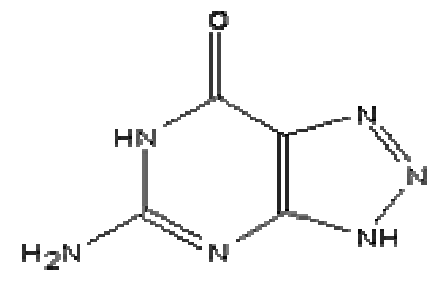

Chloral hydrate<smiles>OC(O)C(Cl)(Cl)Cl</smiles>

Topas<smiles>c1ccc2[nH]c(-c3cscn3)nc2c1</smiles>

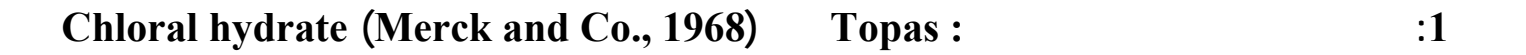

.(King et al., 2006) ن 8- Azaguanine، (Green and Prout,1985)

لذلك فلن الدرلمة الحالية تتهف اله عزل طأرات مقاومة مستحثة بأثبعة اللثهس من الفرر Alternaria alternata ومقاومة

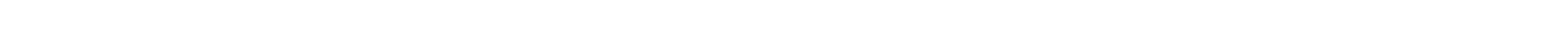

\section{المواد طرلق الهل}

لجريت تجارب البهث على النط البري من النطر Alternaria alternata الذي مّ الحصول عليه من د. ورقاءسعيد قلم/ جلمعة الموصل/كلية العلوم /علوم الحيلة.

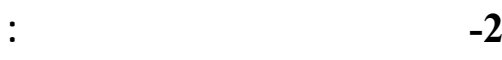

استعمل الوسط الزرعي (Potato Sucrose gar, PSA) (Pitt and Hocking, 2009) لتنمية الفطر وللحصول على كمية كبيرة من الكونيدات كما اعتمد وسط الحد الأنف(Caten, 1979) (Minimal Medium,M) القيلس التركيز المثط الانف المواد الكيميائية قيد الدرلسة وكذك لعزل الطافرات المقاومة لتك المواد ولختبار المقاومة العرضية بين نلك الطافرات. كما اضضف الملح (PSAD, MD)(الل تلك الأوسط ليصح كل منهما (Mackintosh and Pritchard,1963) (Sodium Doxeycholate, D) قعلى التوالي وذك بتركيز 400 مايكروغرلماهل للحصول على مستعمرات مفررة ولتسهيل عملية عزل الطافرات المقاومة.

3- 3تحضير 3واد الاختبار:

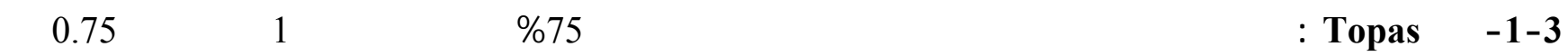

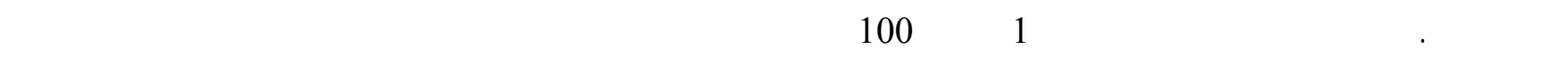
Sharp and تخفيف المحلول الخزين مرة لخرى ليصح التركيز النهائي المحلوله الخزين 750ميكروغرله المل، (للثركة المنتجة) 


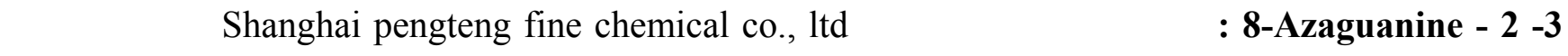

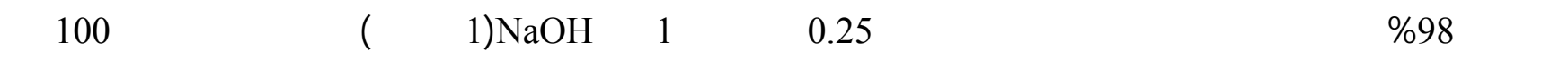
ليصح محلولها الخزين بتركيز 2500مايكرو غرام /لمل(Hoffman and Malling,1974). 3-3-3 - 3loral hydrate بتركيز 100 ملي مولر وقد زونا بها مشكوراً المستاذ الكتورساهي جواد ضالحي /كلية العلوم /قسم علوم الحيلة.

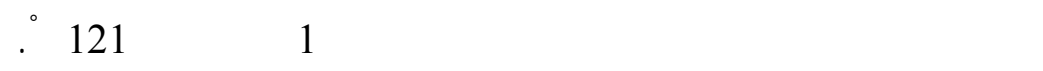
4-قيلس التركيز المثط الأف:

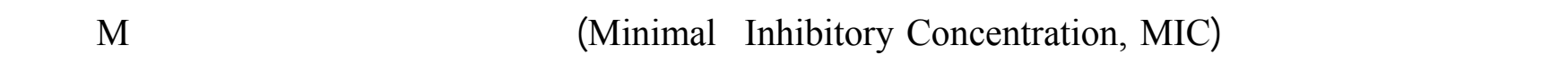

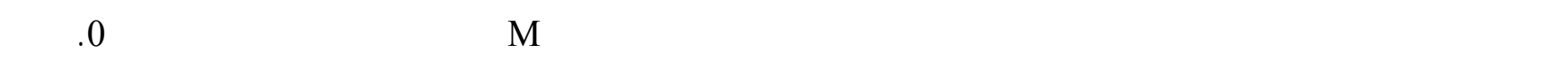

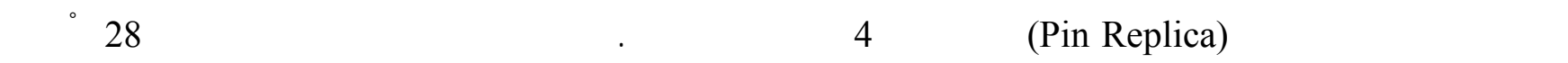
الانتهاء aن التحضن جرى عسلب النسبة المئوية للتببط من خلل مسلب متوسط القار المستعمرات بوجود المادة الكيميائية او عمم وجودها للم مكرر وكما في المعادلة الاتية :

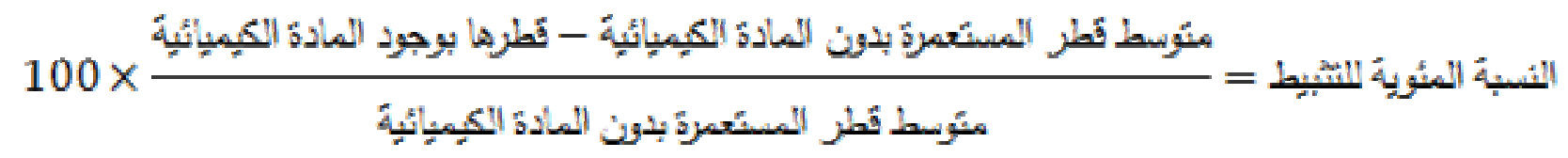

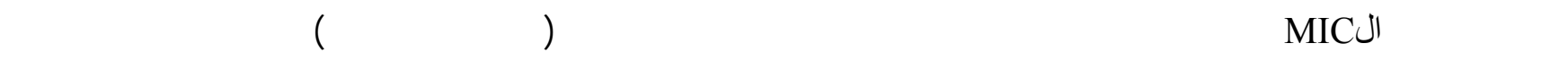
المقاومة.

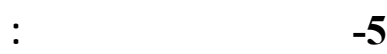

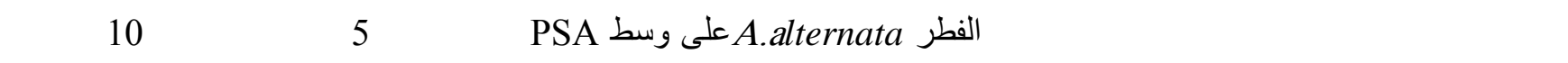

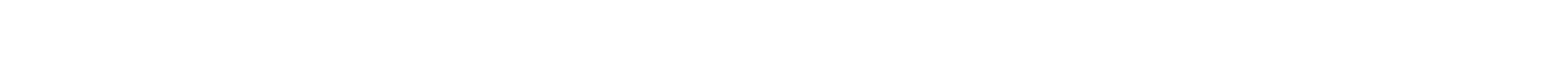

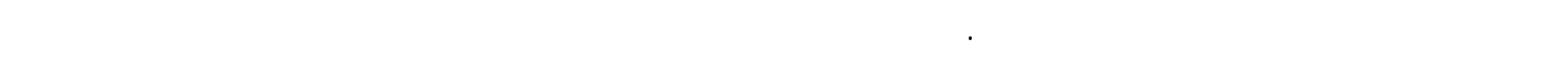

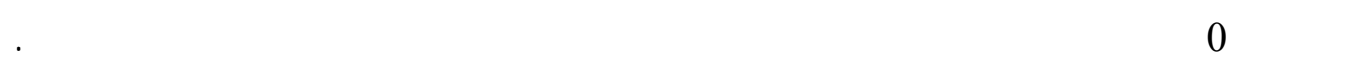
6 لجريت هذه التجربة في وسطشهر تموز ولستمرت اله الولشهر لب في منتصف النهار اذ كلنت درجة الحرارة 40 -45 وذلك من

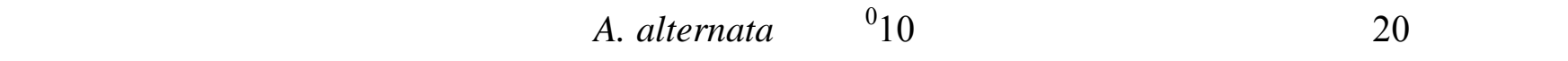

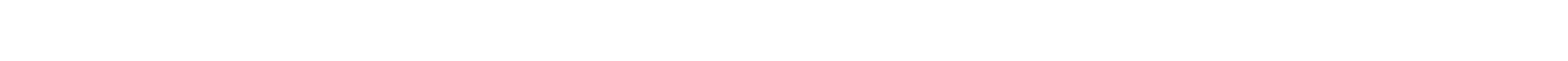
فترة ؤلخرى بلستعمل ماصة معقمة، تمت هذه المعلملة لفترات تعريض مختلفة (20،20،40،60،

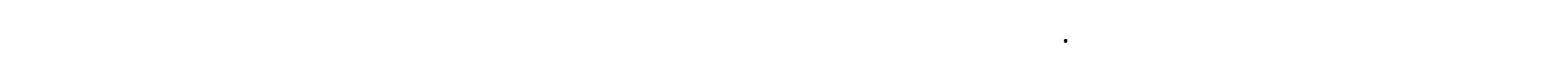

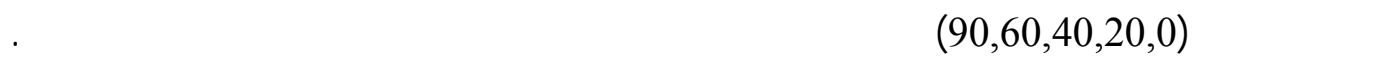

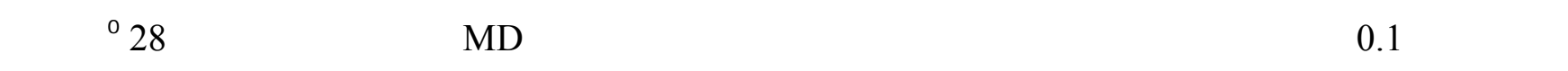

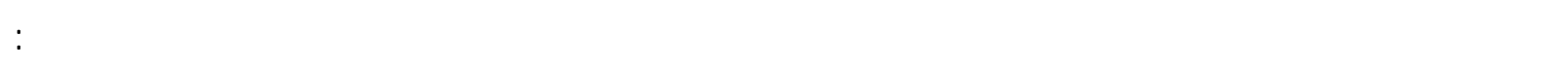
شدة القل = 100 - العدد الهي للأوراد النجية 


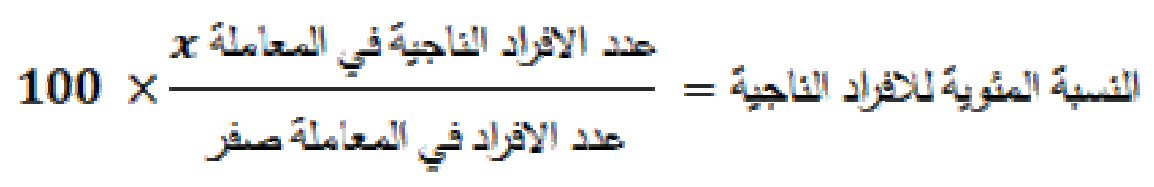
اذ تشيرx اله فترة النعريض للمأشعة.

: 7

مث تعريض العاق الكونيدي ني التخفي 100 وذلك كما ورد في (الجل، 1990) للطرر

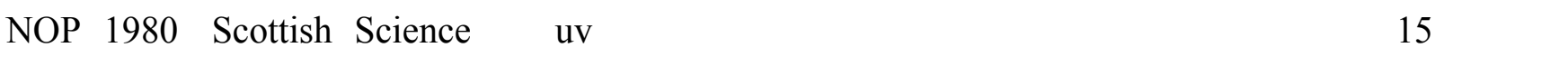
والمجهز منشركة Philip Harris الانكليزية ( اذ يطلق لشعة غالبيتها عندطول موجي 253.7 نانوميتر) عن بعد 10سم عن

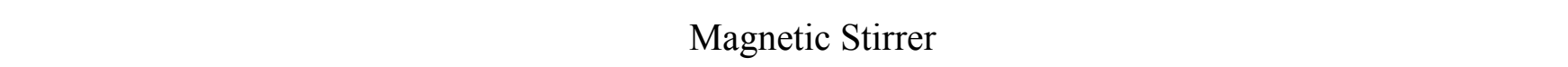

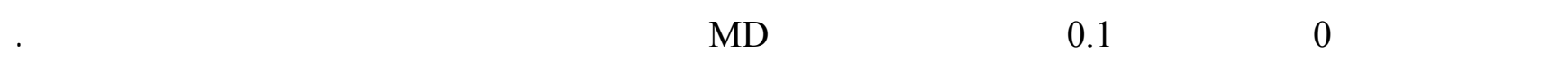
8- عزل اللالفرات المقاومة:

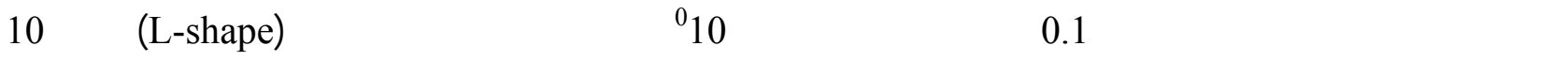

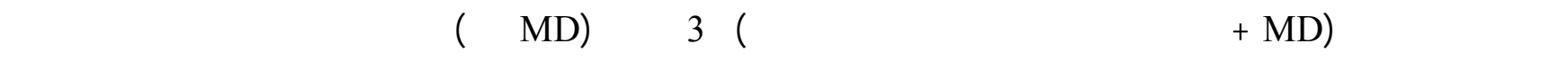

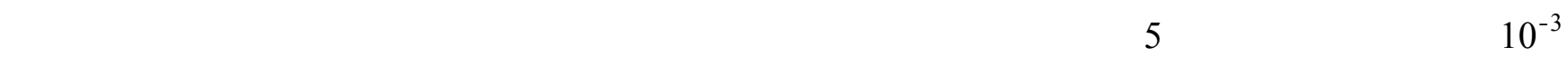

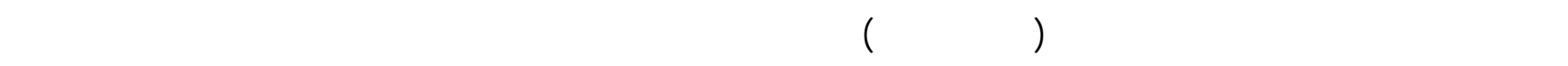

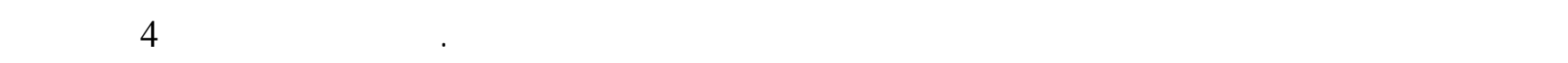

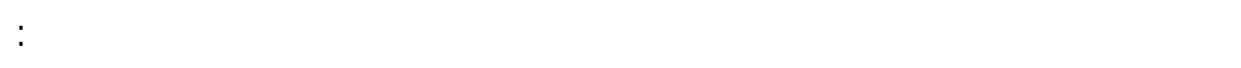
حجم العشيرة المسوحة المتقوعمعل عدد المستعمرات في الطبق الولحدمن MD × مقلوب التخفي ×10

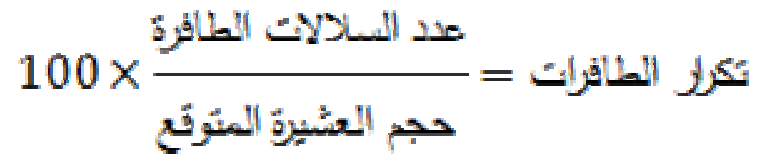

9- الختبار المقاومة المرضية:

قم لختبار المقاومة العرضية للسلالات المقاومة للققاقير الثلاثة المستعملة في البهث من خلالل تلقيح الطافرات المقاومة للكل كقار على وسط يحوي التركيز القالل من العقار الاخر فضلًا عن تلقيح للسلالة البرية غير القادرة على النمو في هذه الترلكيز للمقارنة.

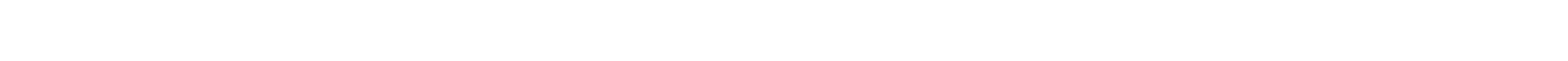
النبة المئوية الطافرات ذات المقاومة العرضية.

\section{النتائج والمناdشة}

التركيز المثا الأف

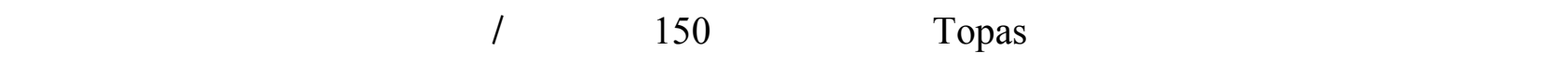
100، أما أنف تركيز قالن لمادة 8-Azaguanine

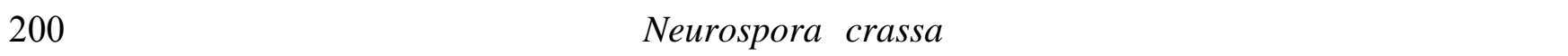


ميكروغرل/مل(Hoffman and Malling,1974). بالنسبة لمادة Chloral hydrate كلن التركيز4.5 ملي مولر هـ -و التركي -ز المثط الأنف وكما في (الجداول 1 و 2 و3) على الترتيب.

الجرل 1: النسبة المئوبة اللتبيا للسلالة AA1 النامية لمدةخسة ليلم علل الومط M الحاوي عل ترلكيزمتصاعة

Tpoas a

\begin{tabular}{|c|c|c|}
\hline النسبة المئوبة اللتثيلا & متومطاقلر المستعمرة لسم & Topas ميكروغرلملل \\
\hline & 1.7 & 0 \\
\hline 11.8 & 1.5 & 3.75 \\
\hline 29 & 1.1 & 11.25 \\
\hline 44.1 & 0.95 & 37.5 \\
\hline 60 & 0.68 & 56.25 \\
\hline 74.7 & 0.43 & 75 \\
\hline 92.3 & 0.13 & 131.25 \\
\hline 100 & 0 & 150 \\
\hline
\end{tabular}

الجرل 2: النسبة المئوبة للتبها للسلالة AA1 النامية لمدة خمسة ليلم عل الومط M الحاوي عل ترلكيزمتصاعةمن ماة 8- Azaguanine

\begin{tabular}{|c|c|c|}
\hline النسبة المئوبة التنبيا & متوطماقر المستعمرة لسم & $\begin{array}{l}\text { 8-Azaguanine } \\
\text { ميكرورلملط }\end{array}$ \\
\hline & 1.7 & 0 \\
\hline 41.1 & 1 & 1.5 \\
\hline 50 & 0.85 & 2 \\
\hline 76.4 & 0.4 & 2.5 \\
\hline 94.1 & 0.1 & 3 \\
\hline 100 & 0 & 3.5 \\
\hline
\end{tabular}

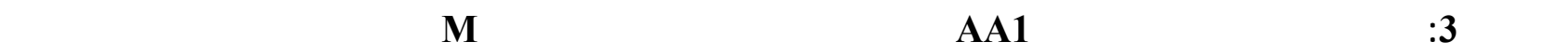
Chloral hydrate مبيد

\begin{tabular}{|c|c|c|}
\hline ain & mM Chloral hydrate \\
\hline 23.5 & 1.3 & 0.5 \\
\hline 41.1 & 1 & 1 \\
\hline 58.8 & 0.7 & 1.5 \\
\hline 92.3 & 0.13 & 3 \\
\hline 96.4 & 0.06 & 3.5 \\
\hline 100 & 0 & 4.5 \\
\hline
\end{tabular}




\section{المعاملة بالثأثعة فوق البفسجية(uv) والثعة للثشس}

جرى تعريض العاق الكونيدي للسلالة البرية Alternaria alternata لأشعة اللشهس وبالظروف المنكورة في طرق العطل وفقرات تعريض مختلفة (0،20،40،60،90) دقيقة وبواقع ثلاث مكررات ومن خلل النتائج الموضحة في (الجدول 4) نجد

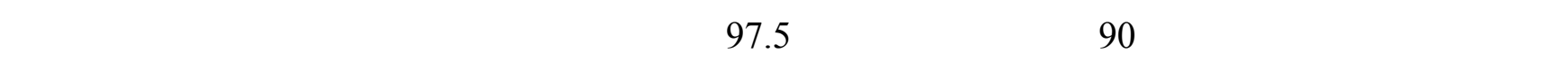
Aspergillus amstelodami الكونيدات قربيها ولأن غاية البهث الحصول على الطافرات المقاومة ولبس قل الخلايا الحية لذك اعتمدت فترة (60) دقيقة وهي قريبباً تمانل اعلىشة قل حثت بلستعمل الاشعة فوق البفسجية عند فترة 30 قققة (الجدول 6) اذ كالت 88.96 فكلما ارتعهت

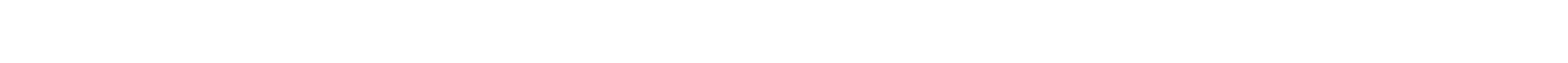

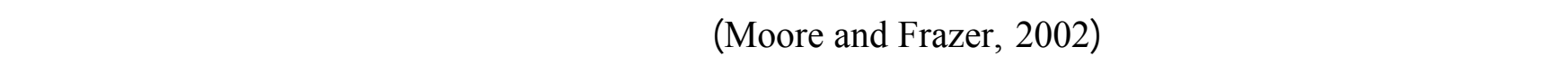

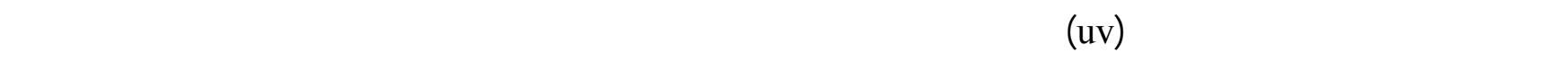

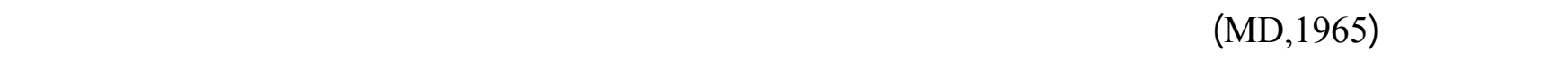

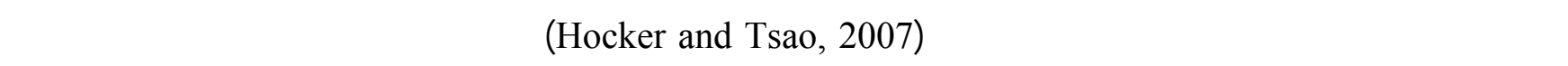

جلد الفئران (Yoon et al., 2009).

تعدد الأشعة فوق البفسجية (Uv) مطفراً فيزيائياً موجباً لقدرته على تكوين ثنائيك الثيلمين المزدوجة في تركيب

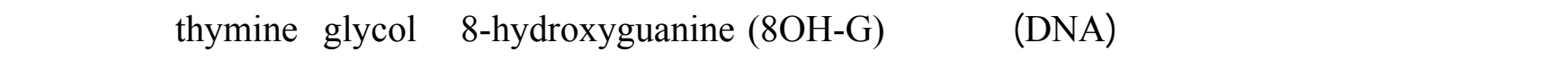
الطفرات الجينية (Ikehata and Ono,2011) ولذك لمستعل هذا المطفر الموجب في هذا البهث للمقارنة فتط مع لشعة الثهن في

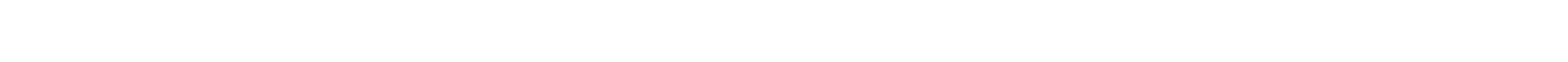

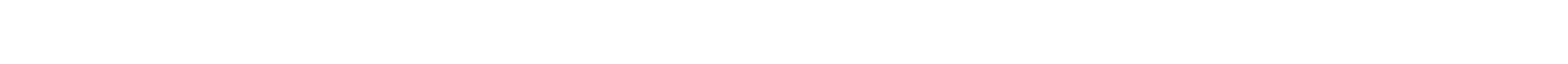

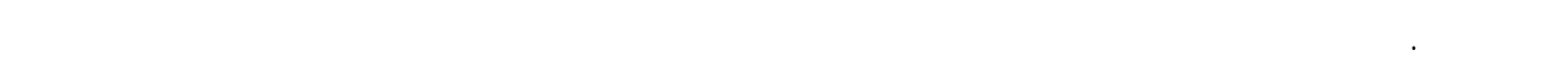

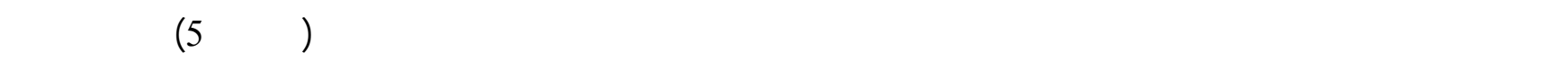

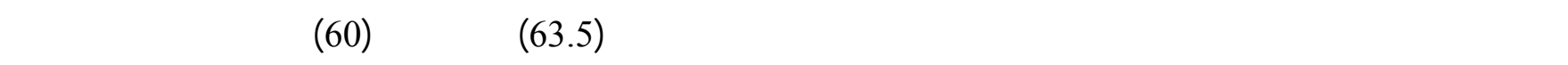

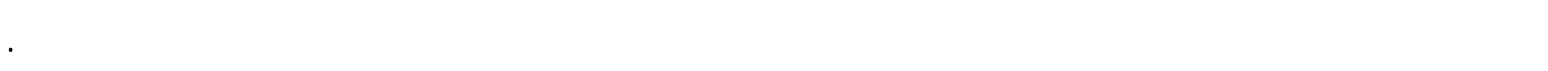

الجرل 4:ششة الفل للعوالق الكونيية الفلر Alternaria alternata المعرضة لأشعة اللشس والغترات زمنيةمختلفة.

\begin{tabular}{|c|c|c|c|c|c|}
\hline \multirow[t]{2}{*}{ شسة القل } & \multirow[t]{2}{*}{ (M) المتوطb } & \multicolumn{3}{|c|}{ مكررات عدد المستهمرات } & \multirow{2}{*}{ فترة النعرضض (ققة) } \\
\hline & & R3 & $\mathbf{R 2}$ & R1 & \\
\hline & 93.6 & 105 & 86 & 90 & صفر \\
\hline $\begin{array}{l}52.9 \\
\end{array}$ & 44 & 53 & 41 & 38 & 20 \\
\hline 76.49 & 22 & 22 & 25 & 19 & 40 \\
\hline 87.6 & 11.6 & 11 & 15 & 9 & 60 \\
\hline 97.5 & 2.3 & 5 & 0 & 2 & 90 \\
\hline
\end{tabular}


هدى وليد هادي

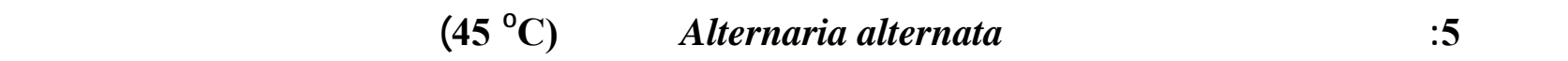

\begin{tabular}{|c|c|c|c|c|c|}
\hline \multirow[t]{2}{*}{ عشعة الفل } & \multirow[t]{2}{*}{ المتوطb (M) } & \multicolumn{3}{|c|}{ مكررل عدد المسنهمرات } & \multirow[t]{2}{*}{ فترة النعرض(قيقة) } \\
\hline & & R3 & R2 & R1 & \\
\hline & 79.66 & 78 & 81 & 80 & صفر \\
\hline 10.9 & 71.0 & 73 & 69 & 71 & 20 \\
\hline 19.3 & 64.33 & 55 & 70 & 68 & 40 \\
\hline 23.9 & 60.6 & 58 & 65 & 59 & 60 \\
\hline 73.3 & 21.33 & 21 & 19 & 24 & 90 \\
\hline
\end{tabular}

الجطل 6:شة الفل للعوالق الكونيية اللعلر Alternaria alternata المعرضة للأشعةفوق البفسجية (uv) ولفترل زمنية مختلة

\begin{tabular}{|c|c|c|c|c|c|}
\hline \multirow[t]{2}{*}{ شسة الفل } & \multirow[t]{2}{*}{ المتوطb (M) } & \multicolumn{3}{|c|}{ مكررات عدد المستهمرات } & \multirow[t]{2}{*}{ فترة المعاملة (فققة) } \\
\hline & & $\mathbf{R 3}$ & $\mathbf{R 2}$ & R1 & \\
\hline & 90.6 & 112 & 93 & 67 & صفر \\
\hline 65.4 & 31.3 & 35 & 27 & 32 & 10 \\
\hline 77.2 & 20.6 & 20 & 19 & 23 & 15 \\
\hline 88.96 & 10 & 9 & 13 & 8 & 30 \\
\hline
\end{tabular}

اللالفرات المقاومة و تكرارلها

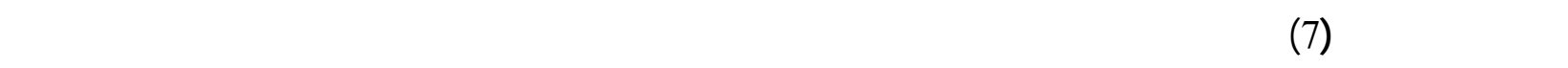

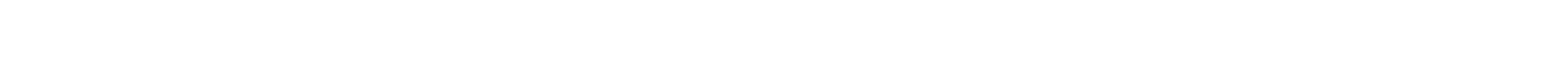

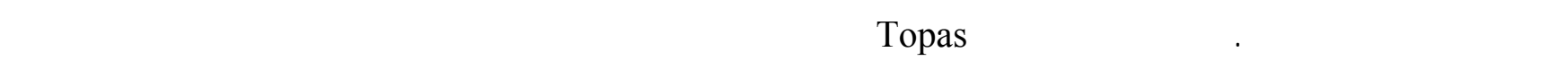

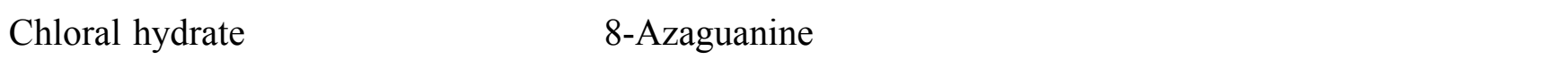

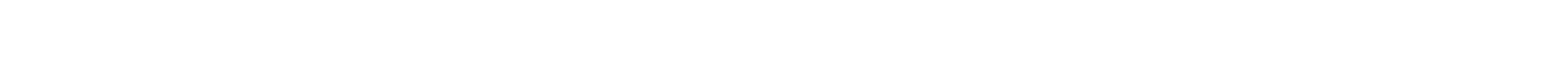

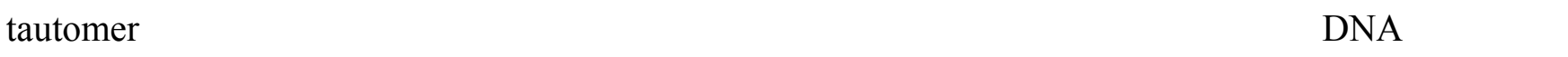

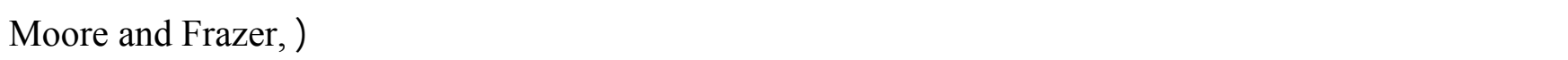

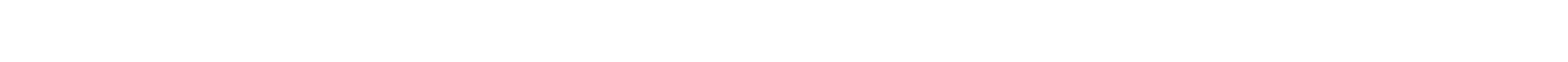

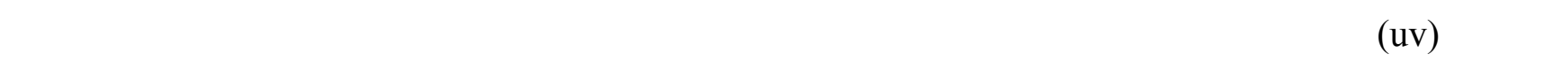

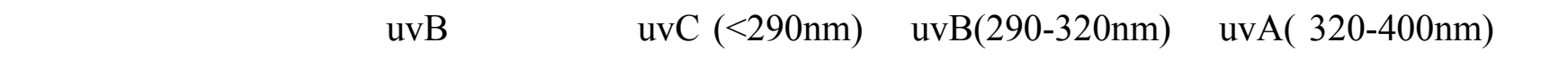
المشعة القدرته على تكوين ثنائيت الثيلمن. وعمليت الاصلاح الناتجة عنها الاطظاء المحثة الطفرات (ROS)، كما يقوم المكون

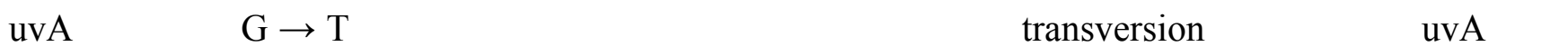


مع uvB في تكوين ما يعرف ببصمة المشعة فوق البفسجية (uv signature) بتكوين انقالات transition فترتبط قاعة

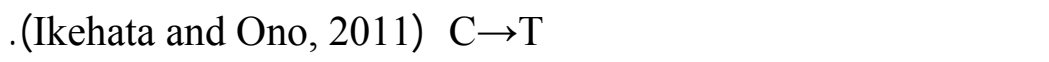
وعند إجراء تحلبل إحصائي جرت مقارنة متوبطلت تكرار الطافرات المقاومة ( للمواد الكيميائية الفة الذكر) التافئية بمتوسطلت تكراراتها المقاومة من المعرضة للأشعة الثهس وقد كلتت قيم t الجدولية اعلى من قيم t المعسوبة عندمستوى لحتمالية 0.01 وكذك عند 0.05، يتبين من ذاك النه لا يوجد فروق معنوية كبيرة بين تلك التكرارات على الرغم من قدرة لشعة اللشهس

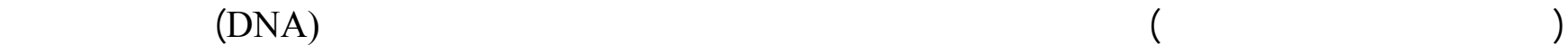
هذه الثشعة ماهي الاخلط من الطاول الموجية المختلفة ذات التأثيرات المختلفة اليضاً (MD, 1965).

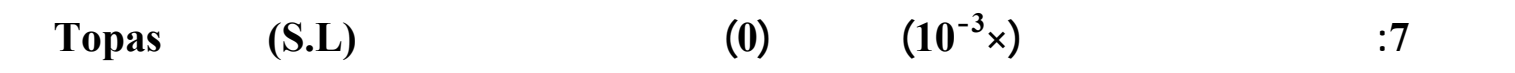
Chloral hydrateg 8-Azaguanine

\begin{tabular}{|c|c|c|c|c|c|c|c|c|c|c|c|}
\hline \multirow[t]{2}{*}{ الميمسة(4) } & \multirow{2}{*}{$\begin{array}{l}\text { الفيلس } \\
\text { (SE) } \\
\end{array}$} & \multirow[t]{2}{*}{\begin{tabular}{|l|} 
المتومط \\
(M)
\end{tabular}} & \multicolumn{3}{|c|}{ (0) } & \multirow{2}{*}{$\begin{array}{c}\text { الفيلسي } \\
\text { الفأ } \\
\text { (SE) }\end{array}$} & \multirow[t]{2}{*}{\begin{tabular}{|l|} 
المتوسط) \\
(M)
\end{tabular}} & \multicolumn{3}{|c|}{ (S.L) } & \multirow[t]{2}{*}{ المالة الكيميائية } \\
\hline & & & R3 & $\mathbf{R 2}$ & R1 & & & R3 & R2 & R1 & \\
\hline $\begin{array}{c}0.8 \\
\text { (ns) }\end{array}$ & 2.7 & 13 & 12 & 9 & 18 & 2.0 & 22 & 19 & 21 & 26 & Topas \\
\hline $\begin{array}{r}2.5 \\
\text { (ns) }\end{array}$ & 1.0 & 16 & 17 & 14 & 17 & 1.2 & 21.3 & 22 & 19 & 23 & 8-Azaguanine \\
\hline $\begin{array}{c}1.9 \\
\text { (ns) }\end{array}$ & 0.33 & 14.7 & 14 & 15 & 15 & 1.4 & 18.7 & 21 & 16 & 19 & Chloral hydrate \\
\hline
\end{tabular}

a sunlight : S.L 0 : معلملة تلفائية بالزرع على الترلكيز القاتلة فتط من المواد الكيميائية.

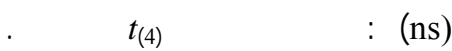

\section{المقاومة العرضية}

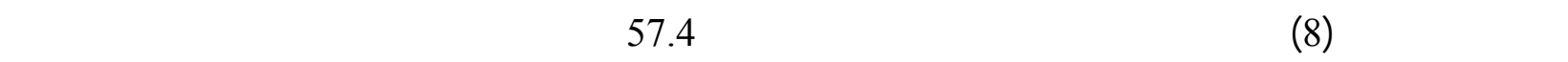
52.4 في الطافرات النقائية وهذا يشير الهى تشابه الهذف الذي تعل عليه كلا الماتين وهو منع تجمبع خيوط المغزل الثناء الاقنسلم

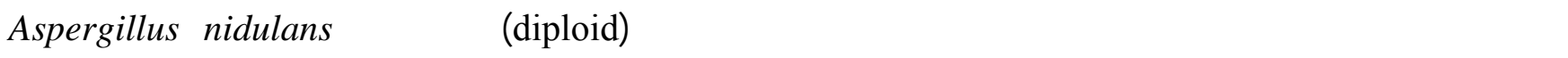

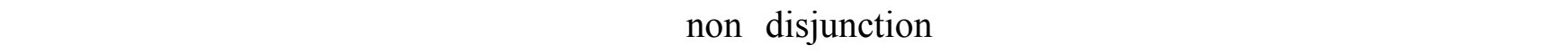
تجاوزت نصف النسبة المئوية بين التوبلس والازلكولنين اذ يل ذلك على تشابه في التركيب الكيميائي اذ ان كلا المركبين يحتويلن على التركيب الحلقي كما مبين في الشكل (1) مما يجعلهما يشتركان في الية القل عبر الغشاء البلازمي وان اي تبنل يحدث في الية

الثل (اي حدوثطفرة) يجعل للسلالة الطافرة مقاومة لكلا المادتين وهذاما لكه (Kanafani and Perfect, 2008).

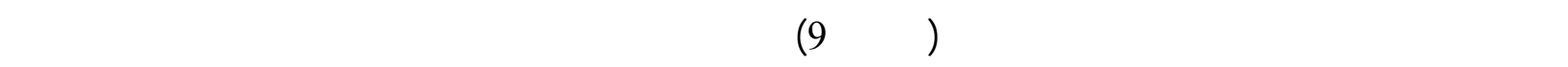

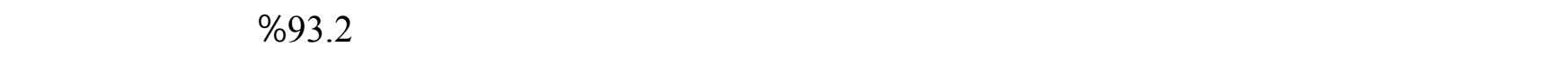


و86.6\% في حالة التائية وهو ققريباً فنس ما اؤثر في المقاومة العرضية لمقاوملت التوبلس مع الازلكوانين (الجدول8) ولكد فنس الإستنتاج للساق. عند إجراء تحلليل إحصائي لمقارنة النبب المئوية لعدد الطافرات المقاومة لماة

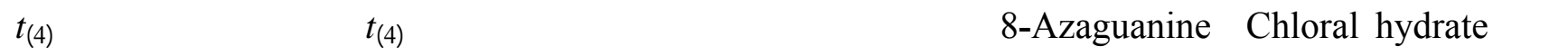

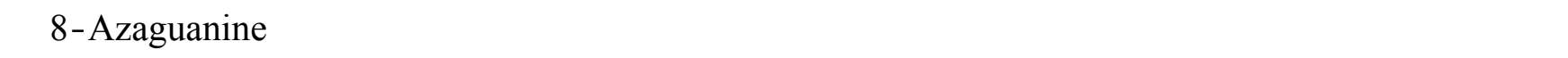

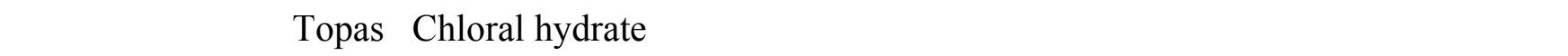
بعدم وجود اي فروق معنوية ربما لان الية حدوث الطفرة المقاومة لهذه للسلالات الطافرة حثت بنفس الالية في حالتيها المقاومة والمعرضة لهذه المواد (Fincham et al.,1979). في (الجدول 10) لم تؤثثر مقاومات الكلورل وجود اي مقاومة عرضية مع الازلكولنين وهو ما حدث فعلاً في لختبار المقاومة العرضية لمقاوملت الازلكوانين مع الكلورل اي الحالة المعلكسة (الجدول 9) نشير هذه النتائج اله الاختلف الكئل الكبير في

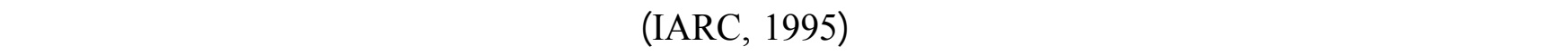

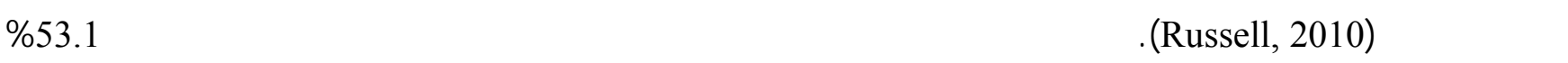
في حالتها التلائية مشابهةً بذك نتيجة الصورة العكسية لمقاوملت التوبلس في لختبار مقاومتها العرضية مع الكلورل (الجدول 8)

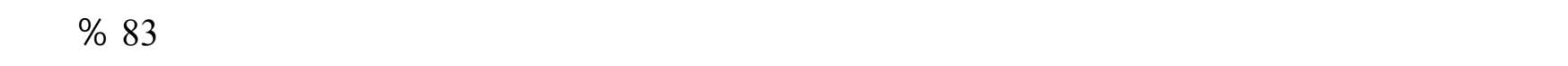

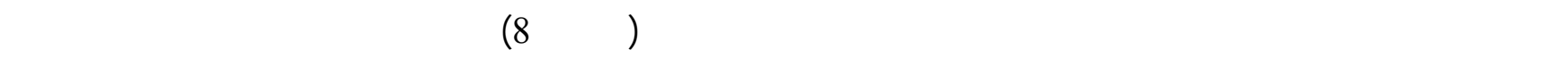
لجراء تحللب لحصائي لمقارنة النسب المئوية للطافرات المقاومة لمادة الكلورل و المعرضة للثالثهس مع التلفائية المقاومة للكلورال

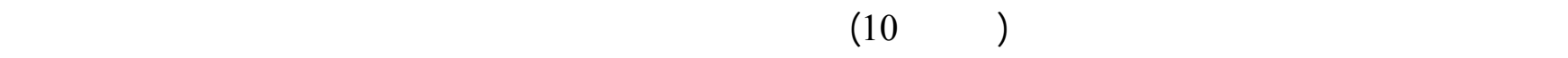

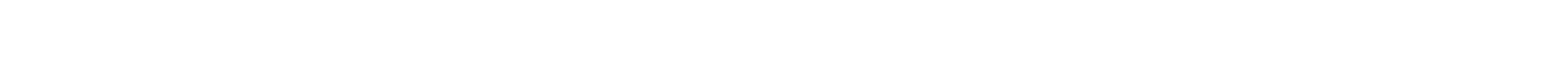
معرضة وليس لدينا قفير لذلك حالياً.

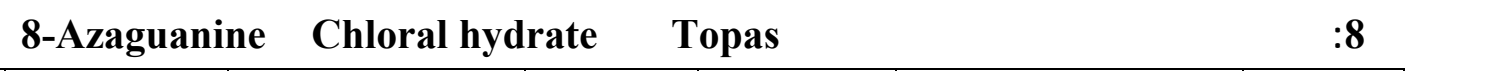

\begin{tabular}{|c|c|c|c|c|c|c|c|c|c|c|}
\hline \multirow{2}{*}{ قالمسمة (4) } & \multirow[t]{2}{*}{$\mathbf{M} \pm \mathbf{S E}$} & \multicolumn{3}{|c|}{$\begin{array}{r}\text { Ea\&.م \% } \\
\text { 8-Azaguanine }\end{array}$} & \multirow{2}{*}{ المسيمة (t) } & \multirow[t]{2}{*}{$\mathbf{M} \pm \mathbf{S E}$} & \multicolumn{3}{|c|}{$\begin{array}{c}\text { caE.p\% } \\
\text { Chloral hydrate }\end{array}$} & \multirow{2}{*}{ الملالهرلت } \\
\hline & & R3 & R2 & R1 & & & R3 & $\mathbf{R 2}$ & R1 & \\
\hline \multirow[t]{2}{*}{$\begin{array}{c}0.1 \\
\text { (ns) }\end{array}$} & $86 \pm 7.8$ & 85 & 73 & 100 & $\begin{array}{l}1.4 \\
\text { (ns) }\end{array}$ & $57.4 \pm 1.8$ & 60 & 58.2 & 54 & S. L \\
\hline & $76.3 \pm 7.5$ & 90 & 75 & 64 & & $52.4 \pm 0.6$ & 53 & 52.9 & 51.2 & 0 \\
\hline
\end{tabular}



عزل بعض الطفورت المقاومة.

الجطل 9: الفقاومة العرضية للالفرل القاومةل Chloral hydrate و 8-Azaguanine مع Topas

\begin{tabular}{|c|c|c|c|c|c|c|c|c|c|c|}
\hline \multirow[t]{2}{*}{ المهسمة } & \multirow[t]{2}{*}{$\mathbf{M} \pm \mathbf{S E}$} & \multirow[t]{2}{*}{ Topas } & \multicolumn{2}{|c|}{ \% م.عمع \% } & \multirow[t]{2}{*}{ فيمة } & \multirow[t]{2}{*}{$\mathbf{M} \pm \mathbf{S E}$} & \multicolumn{3}{|c|}{$\begin{array}{c}\text { ع.ع.م\% } \\
\text { Chloral hydrate }\end{array}$} & \multirow{2}{*}{ الالفرات } \\
\hline & & & R2 & R1 & & & R3 & $\mathbf{R 2}$ & R1 & \\
\hline \multirow[t]{2}{*}{ (ns) 1.5} & $93.2 \pm 1.9$ & 96.5 & 93 & 90 & \multirow[t]{2}{*}{${ }^{*} 2.9$} & $2.3 \pm 0.9$ & 1 & 2 & 4 & S. L \\
\hline & $86.6 \pm 0.9$ & 86.5 & 88.2 & 85 & & $0 \pm 0.0$ & 0 & 0 & 0 & 0 \\
\hline
\end{tabular}

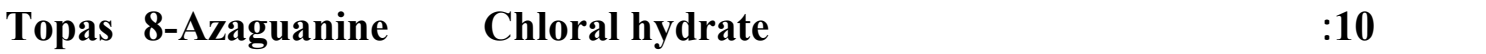

\begin{tabular}{|c|c|c|c|c|c|c|c|c|c|c|}
\hline \multirow{2}{*}{ المهسمة } & \multirow[t]{2}{*}{$\mathbf{M} \pm \mathbf{S E}$} & \multicolumn{3}{|c|}{$\begin{array}{c}\text { Topas } \% \\
\text { Topas }\end{array}$} & \multirow{2}{*}{ قاليمة } & \multirow[t]{2}{*}{$\mathbf{M} \pm \mathbf{S E}$} & \multicolumn{3}{|c|}{$\begin{array}{c}\text { ع.ع\% } \\
\text { 8-Azaguanine }\end{array}$} & \multirow{2}{*}{ الالفرل } \\
\hline & & R3 & R2 & R1 & & & R3 & $\mathbf{R 2}$ & R1 & \\
\hline \multirow[t]{2}{*}{${ }^{*} 9.0$} & $83 \pm 0.6$ & 84.0 & 83.1 & 81.9 & \multirow{2}{*}{$\begin{array}{r}1.5 \\
\text { (ns) }\end{array}$} & & 0 & 0 & 2 & S. L \\
\hline & $53.1 \pm 1.7$ & 53.5 & 55.8 & 50 & & & 0 & 0 & 0 & 0 \\
\hline
\end{tabular}

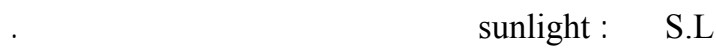

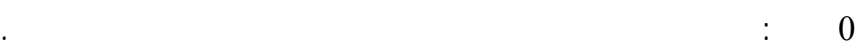

\% م.ع : النسبة المئوية الطافرات ذات المقاومة العرضية التركية

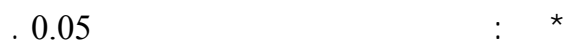

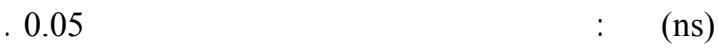

\section{المصارر العربية}

الجلف، بهروز محمود لمين (1990). درلسة وراثية لبعض مقاوملت المايتوز في الفطر Aspergillus amstelodami، بسالة ملجستير، كلية العلوم، العراق.

العال، خالد محمد (2006) . مبيدات الآفت . مفاهيم لسلسية ودورها في المجالين الزراعي والصحي. مكلية الزراعة ـ جلمعة بغداد. جرجيس، رافعة قادربسعيد، جيهلن موفق (2005). التأثير الظفيري لأشعة الثهس في النطر لسبرجلس لم ستيلودلمي. مجل ـة

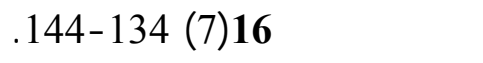

\section{المصادر لأجنبية}

Caten, C.E. (1979). Genetical determination of conidial color Aspergillus heterocaryoticus and relationship of this species to Aspergillus amstelodami. Trans. Br. Mycol. Soc.,73, 65-74.

Crebelli, R.; Conti, L.; Conti, G.; Carere, A. (1991). In vitro studies with nine known or suspected spindle poisons :result in test for chromosome malsegregation in Aspergillus nidulans. J. Mutag.,6,131-136.

Ellis, M.B. (1976). " More Dematiaceous Hyphomycetes". Common Wealth Agriculture Bureau, Kew, England.

Fincham, J.R.S.; Day, P.R.; Radford, A. (1979). "Fungal Genetics". Blackwell, Oxford.

Gehmann, K.; Nyfeler, R.; Leadbeater, A.J.; Nevill, D.J.; Sozzi, D. (1990). A new phenylpyrrole fungicide for broad-spectrum disease control .Bright on Crop Protection Conference, Pests and Diseases, 2, 399-406.

Green, T.; Prout, M.S. (1985). Species differences in response to trichloroethylene. II. Biotransform- 


$$
\text { هرى وليد هادي }
$$

ation in rats and mice. Toxicol Appl Pharmacol. 79, 401-11.

Harding, W.C. (1980). Pesticide Profiles, part two fungicides and nematicides. Univ.Maryland, Coop.Ext. Service Bull.

Hocker, T.; Tsao, H. (2007). Ultraviolet radiation and melanoma: a systematic review and analysis of reported sequence variants. Hum. Mutat 28, 578-588.

Hoffman, G.R.; Malling, H.V. (1974). Mutants of Neurospora crassa resistant to 8azaguanine. J. Gener. Microbiol. 83, 319-326.

Ikehata, H.; Ono, T. (2011). The mechanisms of uv mutagenesis. J. Radiat. Res., 52,115- 125.

International Agency for Research on Cancer (IARC) (1995). IARC Monographs on the Evaluation of Carcinogenic Risks to Humans. Chloral and Chloral Hydrate, pp. 245-269, Vol. 63, IARC, Lyon, France.

Kanafani, Z.A.; Perfect, J.R. (2008). Resistance to antifungal agents: mechanisms and clinical impact. Clin. Infect. Dis. 46, 120-128.

King, R.C.; Stansfield, W.D.; Mulligan, P.K. ( 2006). "A Dictionary of Genetics". Oxford University Press, Inc.

Leroux, P.; Chapeland, F.; Desbrosses, D.; Gredt, M. (1999). Patterns of cross resistance to fungicides in Botryotinia fuckeliana (Botrytis cinrea) isolates from French vineyards. Crop Protec.,18, 687-697.

Mackintosh, M.E.; Pritchard, R.H. (1963). The production and replica plating micro-colonies of Aspergillus nidulans. Genet. Res., 4, 320-322.

MD, S.M. (1965). Composition of sunlight and of a number of ultraviolet lamps Arch. Dermatol. 91, 495-496.

Merck and Co., Inc. (1968). The Name, Chemical Identity, and Composition of the Pesticide Chemical: [Thiabendazole]. (Compilation; unpublished study received Aug 28, 1970 under 1F1031; CDL:093340-B).

Moore, D.; Frazer, L.N. (2002). "Essential Fungal Genetics". Springer- Verlag, New York.

Pantazopoulou, A.; Diallinas, G. (2007). Fungal nucleobase transporter. FEMS Microbiol. Rev. 31,657675.

Pitt, J.I.; Hocking, A.D. (2009). "Fungi and Food Spoilage". Springer, New York. pp. 21-57.

Pose-Juan, E.; Rial-Otero, R.; Lopez-Periago, J.E. (2010). Sorption of penconazole applied as a commercial water-oil emulsion in soils devoted to vineyards. J. Haz.Mat., 182,136-143.

Prasad, V.; Upadhyay, R.S. (2010). Alternaria alternate f. sp. Lycopersici and its toxin trigger production of $\mathrm{H}_{2} \mathrm{O}_{2}$ and ethylene in Tomato. J. Plant. Pathol., 92, 103-108.

Reuveni,R.; Dor, G.; Reuveni, M. (1998). Local and systemic control of powdery mildew (Leveillula taurica) on pepper plants by foliar spray of mono-potassium phosphate. Crop Protec.,17,703-709.

Russell, P.J. (2010). "Genetics". Benjamin, San Francisco.

Smith, R.L.; Harrill, J.C. (1968).Incorporation of 8-azaguanine and guanine by Cladosporium resinae.Appl.Microbiol. 10,1454-1456.

Yoon, J., Prakash, L.; Prakash, S. (2009) Highly error-free role of DNA polymerase $\eta$ in the replicative bypass of uv-induced pyrimidine dimers in mouse and human cells. Proc. Natl. Acad. Sci. USA 106, 18219-18224. 